\title{
Dissipation Limit for the Compressible Navier-Stokes to Euler Equations in One-Dimensional Domains
}

\author{
Shangfei Cui \\ College of Science, University of Shanghai for Science and Technology, Shanghai, China \\ Email: shangfeicui@163.com
}

How to cite this paper: Cui, S.F. (2018) Dissipation Limit for the Compressible Navier-Stokes to Euler Equations in OneDimensional Domains. Journal of Applied Mathematics and Physics, 6, 2142-2158. https://doi.org/10.4236/jamp.2018.610180

Received: October 2, 2018

Accepted: October 26, 2018

Published: October 29, 2018

Copyright $\odot 2018$ by author and Scientific Research Publishing Inc. This work is licensed under the Creative Commons Attribution International License (CC BY 4.0).

http://creativecommons.org/licenses/by/4.0/

\begin{abstract}
We prove that as the viscosity and heat-conductivity coefficients tend to zero, respectively, the global solution of the Navier-Stokes equations for one-dimensional compressible heat-conducting fluids with centered rarefaction data of small strength converges to the centered rarefaction wave solution of the corresponding Euler equations uniformly away from the initial discontinuity.

\section{Keywords}

Compressible Navier-Stokes Equations, Vanishing Viscosity Limit, Rarefaction Waves, Euler Equations
\end{abstract}

\section{Introduction and the Main Result}

We study the asymptotic behavior, as the viscosity and heat-conductivity go to zero, respectively, of solutions to the Cauchy problem for the Navier-Stokes equations for a one-dimensional compressible heat-conducting fluid (in Lagrangian coordinates):

$$
\left\{\begin{array}{l}
v_{t}-u_{x}=0 \\
u_{t}+p_{x}=\varepsilon\left(\frac{u_{x}}{v}\right)_{x}, \\
\left(e+\frac{u^{2}}{2}\right)_{t}+(u p)_{x}=\kappa\left(\frac{\theta_{x}}{v}\right)_{x}+\varepsilon\left(\frac{u u_{x}}{v}\right)_{x},
\end{array}\right.
$$

with (discontinuous) initial data

$$
(u, v, e)(x, 0)=\left(u_{0}, v_{0}, e_{0}\right)(x), x \in R,
$$

where $v, u, \theta, p=p(e, v)$ and $e$ denote the specific volume, the velocity, the 
temperature, the pressure and the internal energy respectively, and $\varepsilon, \kappa$ are the viscosity and heat conductivity coefficients, respectively. At infinity, the initial data $u_{0}, v_{0}, e_{0}$ are assumed to satisfy

$$
\lim _{x \rightarrow \pm \infty}\left(u_{0}, v_{0}, e_{0}\right)(x)=\left(u_{ \pm}, v_{ \pm}, e_{ \pm}\right)
$$

where $u_{ \pm}, v_{ \pm}$and $e_{ \pm}$are given constant states.

The system (1.1), describing the motion of the fluid, is the conservation laws of mass, momentum and energy.

The asymptotic behavior of viscous flows, as the viscosity vanishes, is one of the important topics in the theory of compressible flows. It is expected that a general weak entropy solution to the Euler equations should be (strong) limit of solutions to the corresponding Navier-Stokes equations with same initial data as the viscosity and heat conductivity tend to zero, respectively.

For the one-dimensional compressible isentropic Navier-Stokes equations

$$
\left\{\begin{array}{l}
v_{t}-u_{x}=0, \\
u_{t}+p(v)_{x}=\varepsilon\left(\frac{u_{x}}{v}\right)_{x},
\end{array}\right.
$$

and the corresponding inviscid $p$-system

$$
\left\{\begin{array}{l}
v_{t}-u_{x}=0 \\
u_{t}+p(v)_{x}=0
\end{array}\right.
$$

the vanishing viscosity limit for the Cauchy problem has been studied by several researchers. In [1] Di Perna uses the method of compensated compactness and established almost everywhere convergence of admissible solutions $\left(u^{\varepsilon}, v^{\varepsilon}\right)$ of (1.4) to an admissible solution of (1.5), provided that $\left(u^{\varepsilon}, v^{\varepsilon}\right)$ is uniformly $L^{\infty}$ bounded and $v^{\varepsilon}$ is uniform bounded away from zero. However, this uniform boundedness is difficult to verify in general, and the abstract analysis in [1] gets little information on the qualitative nature of the viscous solutions. In [2] Hoff and Liu investigate the inviscid limit problem for (1.4) in the case that the underlying inviscid flow is a single weak shock wave, and they show that solutions of the compressible Navier-Stokes equations with shock data exist and converge to the inviscid shocks, as viscosity vanishes, uniformly away from the shocks. Based on [2] [3], Xin in [4] shows that the solution to the Cauchy problem for the system (1.4) with weak centered rarefaction wave data exists for all time and converges to the weak centered rarefaction wave solution of the corresponding Euler equations, as the viscosity tends to zero, uniformly away from the initial discontinuity. Moreover, for a given centered rarefaction wave to the Euler equations with finite strength, he constructs a viscous solution to the compressible Navier-Stokes system with initial data depending on the viscosity, such that the viscous solution approaches the centered rarefaction wave as the viscosity goes to zero at the rate $|\ln \varepsilon| \varepsilon^{1 / 4}$ uniformly for all time away from $t=0$. In the vanishing viscosity limit, the Prandtl boundary layers (characteristic boundaries) are studied for the multidimensional linearized compressible Navier-Stokes equ- 
ations by using asymptotic analysis in [5] [6] [7], while the boundary layer stability in the case of non-characteristic boundaries and one spatial dimension is discussed in [8] [9]. We mention that there is an extensive literature on the vanishing artificial viscosity limit for hyperbolic systems of conservation laws, see, for example, [1] [3] [10]-[19], also cf. the monographs [20] [21] [22] and the references therein. We also mention that the convergence of 1-d Broadwell model and the relaxation limit of a rate-type viscoelastic system to the isentropic Euler equations with centered rarefaction wave initial data are studied in [23] [24], respectively. And, in [25], the solution of the Navier-Stokes equations for one-dimensional compressible heat-conducting fluids with centered rarefaction data of small strength had been proved exist globally in time, and moreover, as the viscosity and heat-conductivity coefficients tend to zero, the global solution converges to the centered rarefaction wave solution of the corresponding Euler equations uniformly away from the initial discontinuity.

However, in those paper, $\kappa$ is generally dependent of $\varepsilon$, while in this paper, we will show the dissipation limit in the case that $\kappa$ and $\varepsilon$ are independent of each other.

Our aim in this paper is to study the relation between the solution $\left(u^{\kappa, \varepsilon}, v^{\kappa, \varepsilon}, e^{\kappa, \varepsilon}\right)(x, t)$ of the Navier-Stokes equations for a compressible heatconducting fluid (1.1) and the solution $(u, v, e)(x, t)$ of the corresponding inviscid Euler equations:

$$
\left\{\begin{array}{l}
v_{t}-u_{x}=0, \\
u_{t}+p_{x}=0, \\
\left(e+\frac{u^{2}}{2}\right)_{t}+(u p)_{x}=0,
\end{array}\right.
$$

with the initial data

$$
(u, v, e)(x, 0)=\left(\tilde{u}_{0}, \tilde{v}_{0}, \tilde{e}_{0}\right)(x), \quad x \in R,
$$

satisfying

$$
\lim _{x \rightarrow \pm \infty}\left(\tilde{u}_{0}, \tilde{v}_{0}, \tilde{e}_{0}\right)(x)=\left(u_{ \pm}, v_{ \pm}, e_{ \pm}\right),
$$

with the same constant states $\left(u_{ \pm}, v_{ \pm}, e_{ \pm}\right)$as in (1.3).

It is convenient to work with the equations for the entropy $s$ and the absolute temperature $\theta$. The second law of thermodynamics asserts that

$$
\theta \mathrm{d} s=\mathrm{d} e+p \mathrm{~d} v .
$$

We assume, as is customary in thermodynamics, that given any two of thermodynamics variables $\rho, e, \theta, s$ and $p$, we can obtain the remaining three variables. If we choose $(v, \theta)$ as independent variables and write $(p, e, s)=(p, e, s)(v, \theta)$, we deduce that

$$
s_{v}(v, \theta)=p_{\theta}(v, \theta), \quad s_{\theta}(v, \theta)=\frac{e_{\theta}(v, \theta)}{\theta}, \quad e_{v}(v, \theta)=\theta p_{\theta}(v, \theta)-p(v, \theta) .
$$

Then, a straightforward calculation gives

$$
s_{t}=\kappa\left(\frac{\theta_{x}}{v \theta}\right)_{x}+\kappa \frac{\theta_{x}^{2}}{v \theta^{2}}+\varepsilon \frac{u_{x}^{2}}{v \theta},
$$


and

$$
\theta_{t}+\frac{\theta p_{\theta}(v, \theta)}{e_{\theta}(v, \theta)} u_{x}=\frac{\kappa}{e_{\theta}(v, \theta)}\left(\frac{\theta_{x}}{v}\right)_{x}+\frac{\varepsilon}{e_{\theta}(v, \theta)} \frac{u_{x}^{2}}{v} .
$$

We may also choose $(v, s)$ as independent variables and write

$$
p=p(v, s), \quad \theta=\theta(v, s) .
$$

Thus, instead of (1.1), we shall study the system $(1.1)_{1},(1.1)_{2}$ and (1.9), or $(1.1)_{1},(1.1)_{2}$ and (1.10). Namely, we shall consider

$$
\left\{\begin{array}{l}
v_{t}-u_{x}=0, \\
u_{t}+p(v, s)_{x}=\varepsilon\left(\frac{u_{x}}{v}\right)_{x}, \\
s_{t}=\kappa\left(\frac{\theta_{x}}{v \theta}\right)_{x}+\kappa \frac{\theta_{x}^{2}}{v \theta^{2}}+\varepsilon \frac{u_{x}^{2}}{v \theta},
\end{array}\right.
$$

with initial data

$$
(u, v, s)(x, 0)=\left(u_{0}, v_{0}, s_{0}\right)(x)= \begin{cases}\left(u_{-}, v_{-}, s_{-}\right), & x<0 \\ \left(u_{+}, v_{+}, s_{+}\right), & x>0\end{cases}
$$

where $u_{ \pm}, v_{ \pm}$and $s_{ \pm}$are the constant states. The corresponding inviscid Euler equations read:

$$
\left\{\begin{array}{l}
v_{t}-u_{x}=0, \\
u_{t}+p(v, s)_{x}=0, \\
s_{t}=0 .
\end{array}\right.
$$

We assume in this paper that the pressure $p$ is a smooth function of its arguments satisfying

$$
p_{v}(v, s)<0<p_{v v}(v, s), \quad v>0 .
$$

Notice that the condition (1.14) assures the system (1.13) has characteristic speeds

$$
\lambda_{1}=-\sqrt{-p_{v}}, \quad \lambda_{2}=0, \quad \lambda_{3}=\sqrt{-p_{v}},
$$

and there are two family of rarefaction waves for the Euler equations (1.13). For illustration, we describe only the 1-rarefaction waves, and thus assume $s^{+}=s_{-} \equiv \bar{s}$. The case for the 3-rarefaction waves can be dealt with similarly.

Suppose the end states $\left(u_{ \pm}, v_{ \pm}, \bar{s}\right)$ can be connected by 1 -rarefaction waves. The centered 1-rarefaction wave connecting $\left(u_{-}, v_{-}, \bar{s}\right)$ to $\left(u_{+}, v_{+}, \bar{s}\right)$ is the self-similar solution $(u, v, s)(x, t)=\left(u^{r}, v^{r}, s^{r}\right)(x / t)$ of (1.13) defined by (see, e.g., [26] [27])

$$
\left\{\begin{array}{l}
s^{r}(\xi)=\bar{s}, \\
u^{r}(\xi)=u_{-}+\int_{v_{-}}^{v^{r}(\xi)} \lambda_{1}(z, \bar{s}) \mathrm{d} z, \\
\lambda_{1}\left(v^{r}, \bar{s}\right)(s, t) \text { increasing in } x, \\
\lambda_{1}\left(v^{r}, \bar{s}\right)(x, t)=-\sqrt{-p_{v}\left(v^{r}(x / t), \bar{s}\right)},
\end{array}\right.
$$


which is uniquely determined by the system (1.13) and the rarefaction wave initial data

$$
\left.(u, v, s)\right|_{t=0}=\left(u_{0}^{r}, v_{0}^{r}, s_{0}^{r}\right)(x)= \begin{cases}\left(u_{-}, v_{-}, \bar{s}\right), & x<0, \\ \left(u_{+}, v_{+}, \bar{s}\right), & x>0 .\end{cases}
$$

For the internal energy $e(v, \theta)$, we assume

$$
e_{\theta}(v, \theta)>0 \text { for } v, \theta>0 .
$$

For the sake of convenience, throughout this paper we denote

$$
\alpha=\left|u_{+}-u_{-}\right|+\left|v_{+}-v_{-}\right| \text {. }
$$

In this paper, we prove that the solution of system (1.11) with the centered rarefaction wave initial data (1.16) of small strength $\alpha$ exists for all time and converges to the centered rarefaction wave of the Euler equation (1.13) as $\kappa, \varepsilon$ tends to zero respectively, uniformly away from the initial discontinuity. More precisely, the main result of this paper reads:

THEOREM 1.1. Let the constant states $\left(u_{ \pm}, v_{ \pm}, \bar{s}\right)$ be connected by a centered 1-rarefaction wave $\left(u^{r}(x / t), v^{r}(x / t), s^{r}(x / t)\right)$ defined by (1.15). Assume that (1.14) and (1.17) hold. Then, for $\alpha$ small enough, the compressible Navier-Stokes equations (1.11) with the rarefaction wave initial data (1.16) have a global piecewise smooth solution $\left(u^{\kappa, \varepsilon}(x, t), v^{\kappa, \varepsilon}(x, t), s^{\kappa, \varepsilon}(x, t)\right)$, such that

1) $u^{\kappa, \varepsilon}, \theta^{\kappa, \varepsilon}$ are continuous for $t>0, v^{\kappa, \varepsilon}$ and $u_{x}^{\kappa, \varepsilon}, v_{x}^{\kappa, \varepsilon}, \theta_{x}^{\kappa, \varepsilon}$ are uniformly Hölder continuous in the set $x<0, t \geq \tau$ and $x>0, t \geq \tau$ for any $\tau>0 ; u_{t}^{\kappa, \varepsilon}, u_{x x}^{\kappa, \varepsilon}, v_{x t}^{\kappa, \varepsilon}, \theta_{t}^{\kappa, \varepsilon}, \theta_{x x}^{\kappa, \varepsilon}$ are Hölder continuous on compact set $(x, t), x \neq 0, t>0$. Moreover, the jumps in $v^{\kappa, \varepsilon}$ at $x=0$ satisfy

$$
\left|\left[v^{\kappa, \varepsilon}(0, t)\right]\right| \leq C_{1} \exp \left(-C_{2} t / \varepsilon\right)
$$

and so does the other jumps, where $C_{1}, C_{2}$ are positive constants independent of $t$ and $\varepsilon$, and [.] denotes jumps in what follows.

2) The solution $\left(u^{\kappa, \varepsilon}, v^{\kappa, \varepsilon}, s^{\kappa, \varepsilon}\right)$ converges to the centered rarefaction wave $\left(u^{r}, v^{r}, s^{r}\right)$ as $\varepsilon \rightarrow 0, \kappa \rightarrow 0$ uniformly away from $t=0$, i.e., for any positive $h$, we have

$$
\lim _{\varepsilon \rightarrow 0, \kappa \rightarrow 0} \sup _{x \in \mathbb{R}, t>h}\left|\left(u^{\kappa, \varepsilon}(x, t), v^{\kappa, \varepsilon}(x, t), s^{\kappa, \varepsilon}(x, t)\right)-\left(u^{r}\left(\frac{x}{t}\right), v^{r}\left(\frac{x}{t}\right), s^{r}\left(\frac{x}{t}\right)\right)\right|=0 .
$$

3) For any fixed $\varepsilon>0$ and $\kappa>0$, the solution $\left(u^{\kappa, \varepsilon}, v^{\kappa, \varepsilon}, s^{\kappa, \varepsilon}\right)$ approaches the centered rarefaction wave $\left(u^{r}, v^{r}, s^{r}\right)$ uniformly as time goes to infinity, i.e.,

$$
\limsup _{t \rightarrow \infty}\left|\left(u_{x \in \mathbb{R}^{1}}^{\kappa, \varepsilon}(x, t), v^{\kappa, \varepsilon}(x, t), s^{\kappa, \varepsilon}(x, t)\right)-\left(u^{r}\left(\frac{x}{t}\right), v^{r}\left(\frac{x}{t}\right), s^{r}\left(\frac{x}{t}\right)\right)\right|=0 .
$$

To prove Theorem 1.1 and to overcome the difficulties induced by non-isentropy of the flow, we shall adapt and modify the arguments in [25], but we do not use the natural scaling argument, and we do not assume that $\kappa=O(\varepsilon)$.

We point out here that in view of Theorem 1.1, an initial jump discontinuity at $x=0$ can be allowed in (1.2). The evolution of this jump discontinuity is an 
important aspect in our analysis. It has been shown in [28] that the discontinuity evolution follows a curve $\dot{x}=-[u] /[v]$ in $x$ - $t$ plane, and the jump discontinuity in $v, u_{x}$ and $\theta_{x}$ decays exponentially in time, while the discontinuity in $u$ and $\theta$ are smoothed out at positive time, see [28] for details. We shall exploit this fact in the proof of Theorem 1.1.

In Section 2 we reformulate the problem and give the proof of Theorem 1.1, while Section 3 is dedicated to the derivation of a priori estimates used in Section 2 .

Throughout this paper, we use the following notation:

$$
\begin{aligned}
& \mathbb{R}^{-}:=(-\infty, 0), \quad \mathbb{R}^{+}:=(0, \infty), \quad\|\cdot\|=\|\cdot\|_{L^{2}(\mathbb{R})},\|\cdot\|_{L^{p}}=\|\cdot\|_{L^{p}(\mathbb{R})}, \\
& \|\cdot\|_{ \pm}^{2} \equiv\|\cdot\|_{L^{2}\left(\mathbb{R}^{-}\right)}^{2}+\|\cdot\|_{L^{2}\left(\mathbb{R}^{+}\right)}^{2}, \quad \int_{ \pm} \cdot \mathrm{d} y \equiv \int_{-\infty}^{0} \cdot \mathrm{d} y+\int_{0}^{\infty} \cdot \mathrm{d} y .
\end{aligned}
$$

\section{Reformulation and the Proof of Theorem 1.1}

In this section, we will reduce the proof of Theorem 1.1 to the nonlinear time-asymptotic stability analysis of rarefaction waves for the system (1.11) under non-smooth perturbations.

First, we derive some necessary estimates on the rarefaction waves of the Euler equations (1.13) based on the inviscid Burgers equation, in particularly, we construct an explicit smooth 1-rarefaction wave which well approximates a given centered 1-rarefaction wave. We start with the Riemann problem for the Burgers equation:

$$
\left\{\begin{array}{l}
w_{t}+\left(\frac{w^{2}}{2}\right)_{x}=0, \\
w(x, 0)=w_{0}^{r}(x),
\end{array}\right.
$$

where $w_{0}^{r}(x)$ is given by

$$
w_{0}^{r}(x)= \begin{cases}w_{-}, & x<0 \\ w_{+}, & x>0,\end{cases}
$$

If $w_{-}<w_{+}$, then the problem (2.1) has the centered rarefaction wave solution $w^{r}(x, t)=w^{r}(x / t)$ given by

$$
w^{r}(x, t)=\left\{\begin{array}{l}
w_{-}, \quad x / t \leq w_{-}, \\
x / t, \quad w_{-} \leq x / t \leq w_{+}, \\
w_{+}, \quad x / t \geq w_{+} .
\end{array}\right.
$$

To construct a smooth rarefaction wave solution of the Burgers equation which approximates the centered rarefaction wave, we set for $\delta>0$,

$$
w_{\delta}(x)=w(\delta x)=\frac{w_{+}+w_{-}}{2}+\frac{w_{+}-w_{-}}{2} \tanh (\delta x)
$$

and for each $\delta>0$, we solve the following initial value problem

$$
\left\{\begin{array}{l}
w_{t}+\left(\frac{w^{2}}{2}\right)_{x}=0, \\
w(x, 0)=w_{\delta}(x) .
\end{array}\right.
$$


Next, we state certain properties that will be used later.

LEMMA 2.1. (S. JIANG [25]) For each $\delta>0$, the problem (2.2) has a unique global smooth solution $w_{\delta}^{r}(x, t)$, such that

1) $w_{-}<w_{\delta}^{r}(x, t)<w_{+}, \partial_{x} w_{\delta}^{r}(x, t)>0$ for $x \in \mathbb{R}, t \geq 0, \delta>0$.

2) For any $1 \leq p \leq \infty$, there is a constant $C(p)$ depending only on $p$, such that

$$
\begin{aligned}
& \left\|\partial_{x} w_{\delta}^{r}(\cdot, t)\right\|_{L^{p}} \leq C(p) \min \left\{\left(w_{+}-w_{-}\right) \delta^{1-1 / p},\left(w_{+}-w_{-}\right)^{1 / p} t^{-1+1 / p}\right\}, \\
& \left\|\partial_{x}^{2} w_{\delta}^{r}(\cdot, t)\right\|_{L^{p}} \leq C(p) \min \left\{\left(w_{+}-w_{-}\right) \delta^{2-1 / p}, \delta^{1-1 / p} \frac{1}{t}\right\}, \\
& \left\|\partial_{x}^{3} w_{\delta}^{r}(\cdot, t)\right\|_{L^{p}} \leq C(p) \min \left\{\left(w_{+}-w_{-}\right) \delta^{3-1 / p}, \delta^{2-1 / p} \frac{1}{t}\right\} .
\end{aligned}
$$

3)

$$
\limsup _{t \rightarrow+\infty}\left|w_{x \in \mathbb{R}}^{r}(x, t)-w^{r}(x, t)\right|=0 .
$$

Now, set $w_{ \pm}=\lambda_{1}\left(v_{ \pm}, \bar{s}\right)$, and we define $V(x, t), U(x, t), S(x, t), \Theta(x, t)$, the smooth approximation $\left(v^{r}, u^{r}, s^{r}, \theta^{r}\right)$, by

$$
\begin{aligned}
& \lambda_{1}(V(x, t), \bar{s})=w_{\delta}^{r}(x, t), \quad U(x, t)=u_{ \pm}+\int_{v_{ \pm}}^{V(x, t)} \sqrt{-p_{v}(z, \bar{s})} \mathrm{d} z, \\
& S(x, t)=\bar{s}, \Theta(x, t)=\theta(V(x, t), \bar{s}) .
\end{aligned}
$$

Then, it is not difficult to see that $V(x, t), U(x, t), S(x, t), \Theta(x, t)$ satisfy

$$
\left\{\begin{array}{l}
V_{t}-U_{x}=0, \\
U_{t}+p(V, \Theta)_{x}=0, \\
S_{t}(V, \Theta)=0, \\
\Theta_{t}+\frac{\Theta p_{\Theta}(V, \Theta)}{e_{\Theta}} U_{x}=0,
\end{array}\right.
$$

and due to Lemma 2.1, the following lemma holds for $V(x, t), U(x, t), S(x, t), \Theta(x, t)$.

LEMMA 2.2. (S. JIANG [25]) The functions $V(x, t), U(x, t), S(x, t)$ and $\Theta(x, t)$ constructed above satisfy:

1) $V_{t}=U_{x}>0$ for all $x \in \mathbb{R}, t \geq 0$.

2) For any $1 \leq p \leq \infty$, there is a constant $C(p)$ depending only on $p$, such that

$$
\begin{gathered}
\left\|\left(V_{x}, U_{x}, \Theta_{x}\right)(\cdot, t)\right\|_{L^{p}} \leq C(p) \min \left\{\alpha \delta^{1-1 / p}, \alpha^{1 / p} t^{-1+1 / p}\right\}, \\
\left\|\left(V_{x x}, U_{x x}, \Theta_{x x}\right)(\cdot, t)\right\|_{L^{p}} \leq C(p) \min \left\{\alpha \delta^{2-1 / p}, \delta^{1-1 / p} \frac{1}{t}\right\}, \\
\left\|\left(V_{x x x}, U_{x x x}, \Theta_{x x x}\right)(\cdot, t)\right\|_{L^{p}} \leq C(p) \min \left\{\alpha \delta^{3-1 / p}, \delta^{2-1 / p} \frac{1}{t}\right\} .
\end{gathered}
$$

3)

$$
\limsup _{t \rightarrow \infty}\left|(V, U, S, \Theta)(x, t)-\left(v^{r}, u^{r}, s^{r}, \theta^{r}\right)(x, t)\right|=0 .
$$


4)

$$
\left|\left(V_{t}, U_{t}, \Theta_{t}\right)(x, t)\right| \leq C\left|\left(V_{x}, U_{x}, \Theta_{x}\right)(x, t)\right| .
$$

Consequently, from Lemmas 2.1 and 2.2, it follows that $(V, U, \Theta)(x, t)$ converges to $\left(v^{r}, u^{r}, \theta^{r}\right)(x, t)$ as $t \rightarrow \infty$.

The proof of Theorem 1.1 is broken up into several steps. We start with the observation that by making use of the smooth rarefaction wave $(V, U, \Theta)(x, t)$ constructed above (e.g. one may take $\delta=1$ ), one can decompose the solution $\left(u^{\kappa, \varepsilon}, v^{\kappa, \varepsilon}, \theta^{\kappa, \varepsilon}\right)$ of (1.1), (1.9) and (1.10) into

$$
(\varphi, \psi, \phi)(x, t)=(v-V, u-U, \theta-\Theta)(x, t), \xi(x, t)=s(x, t)-\bar{s} .
$$

Substituting the above decomposition into (1.1), (1.9) and (1.10), we obtain the system for the functions $\varphi, \psi, \phi, \xi$ :

$$
\left\{\begin{array}{l}
\varphi_{t}-\psi_{x}=0, \\
\psi_{t}+(p(v, \theta)-p(V, \Theta))_{x}=\varepsilon\left(\frac{u_{x}}{v}\right)_{x}, \\
\phi_{t}+\frac{\theta p_{\theta}(v, \theta)}{e_{\theta}(v, \theta)} \psi_{x}+\left(\frac{\theta p_{\theta}(v, \theta)}{e_{\theta}(v, \theta)}-\frac{\Theta p_{\Theta}(V, \Theta)}{e_{\Theta}(V, \Theta)}\right) U_{x} \\
=\frac{\kappa}{e_{\theta}(v, \theta)}\left(\frac{\theta_{x}}{v}\right)_{x}+\frac{\varepsilon}{e_{\theta}(v, \theta)} \frac{u_{x}^{2}}{v}, \\
\xi_{t}=\kappa\left(\frac{\theta_{x}}{v \theta}\right)_{x}+\kappa \frac{\theta_{x}^{2}}{v \theta^{2}}+\varepsilon \frac{u_{x}^{2}}{v \theta},
\end{array}\right.
$$

with initial data

$$
(\varphi, \psi, \phi, \xi)(x, 0)=\left(\varphi_{0}, \psi_{0}, \phi_{0}, \xi_{0}\right) \equiv\left(v_{0}-V_{0}, u_{0}-U_{0}, \theta_{0}-\Theta_{0}, s_{0}-\bar{s}\right),
$$

where $\left(\varphi_{0}, \psi_{0}, \phi_{0}, \xi_{0}\right)$ and its derivatives are sufficiently smooth away from $x=0$ but up to $x=0$ and $\left(\varphi_{0}, \psi_{0}, \phi_{0}, \xi_{0}\right) \in L^{2}(\mathbb{R}), \varphi_{0 x} \in L^{2}\left(\mathbb{R}^{-}\right) \cap L^{2}\left(\mathbb{R}^{+}\right)$.

We shall show that the Cauchy problem (2.9), (2.10) possesses a unique global solution $(\varphi, \psi, \phi, \xi)(x, t)$ in the same function class as for $\left(u^{\kappa, \varepsilon}, v^{\kappa, \varepsilon}, \theta^{\kappa, \varepsilon}\right)$ in Theorem 1.1. Moreover, $(\varphi, \psi, \phi)$ goes to zero uniformly as $t \rightarrow 0$. This convergence then yields Theorem 1.1 due to Lemmas 2.1 and 2.2.

LEMMA 2.3. (Hoff [28]) Suppose that $N(0)$ is suitably small so that there exist two positive constants $\underline{v}$ and $\bar{v}$ with $\underline{v} \leq v_{0}^{1}(x) \leq \bar{v}$ for all $x \in \mathbb{R}$. Then, there is a constant $T>0$, such that the Cauchy problem (2.9), (2.10) has a solution $(\varphi, \psi, \phi)$ on $R \times[0, T]$ in the same function class as for $\left(u^{\kappa, \varepsilon}, v^{\kappa, \varepsilon}, \theta^{\kappa, \varepsilon}\right)$ in Theorem 1.1. Moreover, $\varphi, \psi, \phi$ satisfy

1) There exists a positive constant $C$, such that

$$
\sup _{t \geq 0}\left(\|(\varphi, \psi, \phi)(t)\|^{2}+\left\|\varphi_{x}(t)\right\|_{ \pm}^{2}\right)+\int_{0}^{T}\left\|\left(\varphi_{x}, \psi_{x}, \phi_{x}\right)(t)\right\|_{ \pm}^{2} \mathrm{~d} t \leq C\left\{N^{2}(0)+\delta^{1 / 4}\right\} .
$$

2) There is a positive constant $C$, such that

$$
\begin{aligned}
& \sup _{0 \leq t \leq T}\left(\left\|\left(\psi_{x}, \phi_{x}\right)(t)\right\|_{ \pm}^{2}+\left\|\left(\psi_{x x}, \phi_{x x}\right)(t)\right\|_{ \pm}^{2}\right)+\int_{0}^{T}\left\|\left(\psi_{x x}, \psi_{x t}, \phi_{x x}, \phi_{x t}\right)(t)\right\|_{ \pm}^{2} \mathrm{~d} t \\
& \leq C\left\{N^{2}(0)+\delta^{1 / 4}\right\} .
\end{aligned}
$$


3) There are constant $C_{1}, C_{2}>0$ independent of $T$, such that

$$
|[p(v, e)]|=\left|\left[\frac{u_{x}}{v}\right]\right| \leq C_{1} \exp \left\{-C_{2} t\right\}
$$

PROPOSITION 2.4. (A priori estimate)Let the assumptions in Lemma 2.3 be satisfied. Assume that the Cauchy problem (2.9), (2.10) has a solution $(\varphi, \psi, \phi)(x, t)$ on $R \times[0, T]$ for some $t>0$, in the same function class as in Lemma 2.3. Denote

$$
N^{2}\left(t_{0}, t\right):=\sup _{t_{0} \leq s \leq t}\left\{\|(\varphi, \psi, \phi)(t)\|^{2}+\left\|\varphi_{x}(t)\right\|_{ \pm}^{2}+\left\|\psi_{x}(t)\right\|_{ \pm}^{2}+\left\|\phi_{x}(t)\right\|_{ \pm}^{2}\right\}, \quad 0 \leq t_{0} \leq t .
$$

Then, there are positive constants $\eta_{1}$ and $C$ independent of $t_{1}$, such that for each fixed $t_{0}$, if

$$
N^{2}\left(t_{0}, t_{1}\right) \leq \eta_{1}
$$

then the following estimates hold

$$
\begin{aligned}
& N^{2}\left(t_{0}, t_{1}\right)+\int_{t_{0}}^{t}\left\|\left(\varphi_{x}, \psi_{x}, \phi_{x}\right)(\hat{t})\right\|^{2} \mathrm{~d} \hat{t} \\
& \leq C N^{2}\left(t_{0}, t_{0}\right)+C \varepsilon \int_{t_{0}}^{t}\left(\left\|\left(\varphi_{x}, \psi_{x}, \psi_{x x}, \phi_{x}, \phi_{x x}\right)\right\|^{2}+\left\|\psi_{x}\right\|\right)(\hat{t}) \mathrm{d} \hat{t} \\
&+C \kappa \int_{t_{0}}^{t}\left(\left\|\left(\varphi_{x}, \phi_{x}\right)\right\|^{2}+\left\|\left(\varphi_{x}, \phi_{x}\right)\right\|\right)(\hat{t}) \mathrm{d} \hat{t}+C \varepsilon+C \kappa+C \delta^{1 / 4}, \\
& \sup _{0 \leq t \leq T}\left\|\left(\psi_{x}, \phi_{x}\right)(t)\right\|_{ \pm}^{2}+\int_{t_{0}}^{t}\left\|\left(\psi_{x x}, \phi_{x x}\right)\right\|_{ \pm}^{2}(\hat{t}) \mathrm{d} \hat{t} \\
& \leq C\left\{\left\|(\varphi, \psi, \phi)\left(t_{0}\right)\right\|_{ \pm}^{2}+\left\|\left(\varphi_{x}, \psi_{x}, \phi_{x}\right)\left(t_{0}\right)\right\|_{ \pm}^{2}+\delta^{1 / 4}\right\} \\
&+C \varepsilon \int_{t_{0}}^{t}\left(\left\|\left(\varphi_{x}, \psi_{x}, \psi_{x x}, \phi_{x}, \phi_{x x}\right)\right\|_{ \pm}^{2}+\left\|\psi_{x}\right\|_{ \pm}\right)(\hat{t}) \mathrm{d} \hat{t} \\
&+C \kappa \int_{t_{0}}^{t}\left(\left\|\left(\varphi_{x}, \phi_{x}\right)\right\|_{ \pm}+\left\|\left(\psi_{x}, \phi_{x}\right)\right\|_{ \pm}^{2}+\left\|\left(\phi_{x}, \phi_{x x}\right)\right\|_{ \pm}^{2}\right)(\hat{t}) \mathrm{d} \hat{t}+C \varepsilon+C \kappa .
\end{aligned}
$$

Proof of Theorem 1.1. By the systems (2.3) and (2.9), Lemma 2.2, Cauchy-Schwarz's and Sobolev's inequalities, we easily find that

$$
\int_{0}^{\infty}\left\|\left(\varphi_{t}, \psi_{t}, \phi_{t}\right)(t)\right\|_{L^{\infty}}^{2} \mathrm{~d} t<\infty
$$

which together with Lemma 2.3 yields $\limsup _{t \rightarrow \infty}|(\varphi(x, t), \psi(x, t), \phi(x, t))| \rightarrow 0$. Hence, In view of Lemma 2.2, we have proved Theorem 1.1.

\section{Uniform a Priori Estimates}

In this section we derive the key a priori estimates given in Proposition 2.4. First, we introduce the normalized entropy:

$$
\begin{aligned}
\eta(v, u, s, V, U, S):= & \left(e(v, \theta)+\frac{u^{2}}{2}\right)-\left(e(V, \Theta)+\frac{U^{2}}{2}\right) \\
& -\{-p(V, \Theta)(v-V)+U(u-U)+\Theta(s-S)\},
\end{aligned}
$$

where we have used the fact that $e_{v}(v, s)=-p(v, \theta), e_{s}(v, s)=0$.

An easy computation implies that $\eta$ satisfies the equation: 


$$
\begin{aligned}
\eta_{t} & +\{(p(v, \theta)-p(V, \Theta)) \psi\}_{x}+\left(\varepsilon \frac{\Theta \psi_{x}^{2}}{v \theta}+\kappa \frac{\Theta \phi_{x}^{2}}{v \theta^{2}}\right) \\
- & U_{x}\left\{p_{V}(V, \Theta) \varphi-p_{\Theta}(V, \Theta) \frac{\Theta p_{\Theta}(V, \Theta)}{e_{\Theta}} \varphi+\frac{\Theta p_{\Theta}(V, \Theta)}{e_{\Theta}} \xi\right\} \\
= & \left(\varepsilon \frac{\psi \psi_{x}}{v}+\kappa \frac{\phi \phi_{x}}{v \theta}\right)_{x}+\left(\varepsilon \frac{\psi U_{x x}}{v}+\kappa \frac{\phi \Theta_{x x}}{v \theta}\right) \\
& +\left(-\varepsilon \frac{\psi \varphi_{x} U_{x}}{v^{2}}-\varepsilon \frac{\psi \psi_{x} V_{x}}{v^{2}}+\varepsilon \frac{2 \phi \psi_{x} U_{x}}{v \theta}-\kappa \frac{\phi \varphi_{x} \Theta_{x}}{v^{2} \theta}+\kappa \frac{\phi \phi_{x} \Theta_{x}}{v \theta^{2}}\right) \\
& +\left(\varepsilon \frac{\phi U_{x}^{2}}{v \theta}-\varepsilon \frac{\psi U_{x} V_{x}}{v^{2}}+\frac{\phi \Theta_{x}^{2}}{v \theta^{2}}-\kappa \frac{\phi \Theta_{x} V_{x}}{v^{2} \theta}-\kappa \frac{\phi \Theta_{x}^{2}}{v \theta^{2}}\right) .
\end{aligned}
$$

Employing (3.1), one has

LEMMA3.1. Suppose that the assumptions of Proposition 2.4 hold. Then,

$$
\begin{aligned}
& \|(\varphi, \psi, \phi)(t)\|^{2}+\int_{t_{0}}^{t}\left(\left\|\sqrt{V_{t}}(\varphi, \phi)\right\|^{2}+\left\|\left(\psi_{x}, \phi_{x}\right)\right\|_{ \pm}^{2}\right)(\hat{t}) \mathrm{d} \hat{t} \\
& \leq C\left(\left\|(\varphi, \psi, \phi)\left(t_{0}\right)\right\|^{2}+N\left(t_{0}, t\right) \int_{t_{0}}^{t}\left\|\phi_{x}\right\|_{ \pm}^{2}(\hat{t}) \mathrm{d} \hat{t}\right)+C \delta^{1 / 4} \\
& +C \varepsilon N\left(t_{0}, t\right)^{2 / 3} \int_{t_{0}}^{t}\left\|\left(\varphi_{x}, \psi_{x}, \phi_{x}\right)\right\|_{ \pm}^{2}(\hat{t}) \mathrm{d} \hat{t} \\
& \quad+C \kappa N\left(t_{0}, t\right)^{2 / 3} \int_{t_{0}}^{t}\left\|\left(\varphi_{x}, \phi_{x}\right)\right\|_{ \pm}^{2}(\hat{t}) \mathrm{d} \hat{t} .
\end{aligned}
$$

PROOF. Integrating (3.1) with respect to $t$ and $x$, we get

$$
\begin{aligned}
& \|(\varphi, \psi, \phi)(t)\|^{2}+\int_{t_{0}}^{t}\left(\left\|\sqrt{V_{t}}(\varphi, \phi)\right\|^{2}+\left\|\left(\psi_{x}, \phi_{x}\right)\right\|_{ \pm}^{2}\right)(\hat{t}) \mathrm{d} \hat{t} \\
& \leq C\left(\left\|(\varphi, \psi, \phi)\left(t_{0}\right)\right\|^{2}+\sum_{j=1}^{j=4} R_{j}\right),
\end{aligned}
$$

where

$$
\begin{aligned}
& R_{1}=\int_{t_{0}}^{t} \int_{ \pm}\left((-(p(v, \theta)-p(V, \Theta)) \psi)_{x}+\left(\varepsilon \frac{\psi \psi_{x}}{v}+\kappa \frac{\phi \phi_{x}}{v \theta}\right)_{x}\right)(x, \hat{t}) \mathrm{d} x \mathrm{~d} \hat{t}, \\
& R_{2}=\int_{t_{0}}^{t} \int_{ \pm}\left(\varepsilon\left|\psi U_{x x}\right|+\kappa\left|\phi \Theta_{x x}\right|\right)(x, \hat{t}) \mathrm{d} x \mathrm{~d} \hat{t}, \\
& R_{3}=\int_{t_{0}}^{t} \int_{ \pm}\left(\varepsilon\left(\left|U_{x} \psi \varphi_{x}\right|+\left|U_{x} \psi_{x} \phi\right|+\left|V_{x} \psi \psi_{x}\right|\right)+\kappa\left(\left|\Theta_{x} \varphi_{x} \phi\right|+\left|\Theta_{x} \phi \phi_{x}\right|\right)\right)(x, \hat{t}) \mathrm{d} x \mathrm{~d} \hat{t}, \\
& R_{4}=\int_{t_{0}}^{t} \int_{ \pm}\left(\left|\phi \Theta_{x}^{2}\right|+\varepsilon\left(\left|U_{x}^{2} \phi\right|+\left|U_{x} V_{x} \psi\right|\right)+\kappa\left(\left|V_{x} \Theta_{x} \phi\right|+\left|\Theta_{x}^{2} \phi\right|\right)\right)(x, \hat{t}) \mathrm{d} x \mathrm{~d} \hat{t} .
\end{aligned}
$$

Recalling the definition of $N\left(t_{0}, t\right)$ and applying Lemma 2.2, for given $\alpha, R_{j}$ can be estimated as follows.

$$
\begin{aligned}
& R_{1}=\int_{t_{0}}^{t}\left(\left[-p(v, \theta)+p(V, \Theta)+\varepsilon \frac{\psi_{x}}{v}\right] \psi+\kappa\left[\frac{\phi \phi_{x}}{v \theta}\right]\right)(\hat{t}) \mathrm{d} x \mathrm{~d} \hat{t}=0, \\
& R_{2} \leq C \int_{t_{0}}^{t} \varepsilon\left(\|\psi\|^{1 / 2}\left\|\psi_{x}\right\|^{1 / 2}\left\|U_{x x}\right\|_{L^{1}}\right)(\hat{t}) \mathrm{d} \hat{t} \\
& \quad+C \int_{t_{0}}^{t} \kappa\left(\|\phi\|^{1 / 2}\left\|\phi_{x}\right\|^{1 / 2}\left\|\Theta_{x x}\right\|_{L^{1}}\right)(\hat{t}) \mathrm{d} \hat{t} \\
& \leq C \int_{t_{0}}^{t} \varepsilon\left(N\left(t_{0}, t\right)\left\|\psi_{x}\right\|^{2}+\left\|U_{x x}\right\|_{L^{1}}^{4 / 3}\right)(\hat{t}) \mathrm{d} \hat{t} \\
& \quad+C \int_{t_{0}}^{t} \kappa\left(N\left(t_{0}, t\right)\left\|\phi_{x}\right\|^{2}+\left\|\Theta_{x x}\right\|_{L^{1}}^{4 / 3}\right)(\hat{t}) \mathrm{d} \hat{t} \\
& \leq C \varepsilon\left(N\left(t_{0}, t\right) \int_{t_{0}}^{t}\left\|\psi_{x}\right\|^{2}(\hat{t}) \mathrm{d} \hat{t}+\delta^{1 / 4}\right) \\
&+C \kappa\left(N\left(t_{0}, t\right) \int_{t_{0}}^{t}\left\|\phi_{x}\right\|^{2}(\hat{t}) \mathrm{d} \hat{t}+\delta^{1 / 4}\right)
\end{aligned}
$$




$$
\begin{aligned}
R_{4} \leq & C \int_{t_{0}}^{t}\left(\|\phi\|^{1 / 2}\left\|\phi_{x}\right\|^{1 / 2}\left\|\Theta_{x}\right\|^{2}\right)(\hat{t}) \mathrm{d} \hat{t} \\
& +C \varepsilon \int_{t_{0}}^{t}\left(\|(\psi, \phi)\|^{1 / 2}\left\|\left(\psi_{x}, \phi_{x}\right)\right\|^{1 / 2}\left\|\left(U_{x}, V_{x}\right)\right\|^{2}\right)(\hat{t}) \mathrm{d} \hat{t} \\
& +C \kappa \int_{t_{0}}^{t}\left(\|(\varphi, \phi)\|^{1 / 2}\left\|\left(\varphi_{x}, \phi_{x}\right)\right\|^{1 / 2}\left\|\Theta_{x}\right\|^{2}\right)(\hat{t}) \mathrm{d} \hat{t} \\
\leq & C \int_{t_{0}}^{t}\left(N\left(t_{0}, t\right)\left\|\phi_{x}\right\|^{2}+\left\|\Theta_{x}\right\|^{8 / 3}\right)(\hat{t}) \mathrm{d} \hat{t} \\
& +C \varepsilon \int_{t_{0}}^{t}\left(N\left(t_{0}, t\right)\left\|\left(\psi_{x}, \phi_{x}\right)\right\|^{2}+\left\|\left(U_{x}, V_{x}\right)\right\|^{8 / 3}\right)(\hat{t}) \mathrm{d} \hat{t} \\
& +C \kappa \int_{t_{0}}^{t}\left(N\left(t_{0}, t\right)\left\|\left(\varphi_{x}, \phi_{x}\right)\right\|^{2}+\left\|\Theta_{x}(\hat{t})\right\|^{8 / 3}\right)(\hat{t}) \mathrm{d} \hat{t} \\
\leq & C\left(N\left(t_{0}, t\right) \int_{t_{0}}^{t}\left\|\phi_{x}\right\|^{2}(\hat{t}) \mathrm{d} \hat{t}+\delta^{1 / 4}\right) \\
& +C \varepsilon\left(N\left(t_{0}, t\right) \int_{t_{0}}^{t}\left\|\left(\psi_{x}, \phi_{x}\right)\right\|^{2}(\hat{t}) \mathrm{d} \hat{t}+\delta^{1 / 4}\right) \\
& +C \kappa\left(N\left(t_{0}, t\right) \int_{t_{0}}^{t}\left\|\left(\varphi_{x}, \phi_{x}\right)\right\|^{2}(\hat{t}) \mathrm{d} \hat{t}+\delta^{1 / 4}\right),
\end{aligned}
$$

and

$$
\begin{aligned}
R_{3} \leq & C \varepsilon \int_{t_{0}}^{t}\left(\|(\psi, \phi, \varphi)\|^{1 / 2}\left\|\left(\psi_{x}, \phi_{x}, \varphi_{x}\right)\right\|^{3 / 2}\left\|\left(U_{x}, V_{x}\right)\right\|\right)(\hat{t}) \mathrm{d} \hat{t} \\
& +C \kappa \int_{t_{0}}^{t}\left(\|(\varphi, \phi)\|^{1 / 2}\left\|\left(\varphi_{x}, \phi_{x}\right)\right\|^{3 / 2}\left\|\Theta_{x}\right\|\right)(\hat{t}) \mathrm{d} \hat{t} \\
\leq & C \varepsilon \int_{t_{0}}^{t}\left\{N\left(t_{0}, t\right)^{2 / 3}\left\|\left(\psi_{x}, \phi_{x}, \varphi_{x}\right)\right\|^{2}+\left\|\left(U_{x}, V_{x}\right)\right\|^{4}\right\}(\hat{t}) \mathrm{d} \hat{t} \\
& +C \kappa \int_{t_{0}}^{t}\left\{N\left(t_{0}, t\right)^{2 / 3}\left\|\left(\varphi_{x}, \phi_{x}\right)\right\|^{2}+\left\|\Theta_{x}\right\|^{4}\right\}(\hat{t}) \mathrm{d} \hat{t} \\
\leq & C \varepsilon\left\{N\left(t_{0}, t\right)^{2 / 3} \int_{t_{0}}^{t}\left\|\left(\psi_{x}, \phi_{x}, \varphi_{x}\right)\right\|^{2}(\hat{t}) \mathrm{d} \hat{t}+\delta^{1 / 4}\right\} \\
& +C \kappa\left\{N\left(t_{0}, t\right)^{2 / 3} \int_{t_{0}}^{t}\left\|\left(\varphi_{x}, \phi_{x}\right)\right\|^{2}(\hat{t}) \mathrm{d} \hat{t}+\delta^{1 / 4}\right\} .
\end{aligned}
$$

where we have used Sobolev's inequality:

$$
\|u\|_{L^{n p /(n-p)}\left(\mathbb{R}^{n}\right)} \leq C\|D u\|_{L^{p}\left(\mathbb{R}^{n}\right)}, 1 \leq p<n \text {, for all } u \in C_{c}^{1}\left(\mathbb{R}^{n}\right)
$$

Hölder's inequality:

$$
\begin{aligned}
& \|u v\|_{L^{1}(U)} \leq\|u\|_{L^{p}(U)}\|v\|_{L^{q}(U)}, \\
& \text { for } 1 \leq p, q \leq \infty, \frac{1}{p}+\frac{1}{q}=1, u \in L^{p}(U), v \in L^{q}(U) .
\end{aligned}
$$

Young's inequality:

$$
\begin{aligned}
& \|u v\|_{L^{1}(U)} \leq \frac{1}{p}\|u\|_{L^{p}(U)}^{p}+\frac{1}{q}\|v\|_{L^{q}(U)}^{q}, \\
& \text { for } 1 \leq p, q \leq \infty, \frac{1}{p}+\frac{1}{q}=1, u \in L^{p}(U), v \in L^{q}(U) .
\end{aligned}
$$

and the following inequality:

$$
\begin{aligned}
\int_{t_{0}}^{t}\left\|\partial_{x}^{i} U(\hat{t})\right\|_{L^{p}}^{a+b} \mathrm{~d} \hat{t} & \leq \sup _{\hat{t} \in\left[t_{0}, t\right]}\left\|\partial_{x}^{i} U(\hat{t})\right\|_{L^{p}}^{a} \int_{t_{0}}^{t}\left\|\partial_{x}^{i} U(\hat{t})\right\|_{L^{p}}^{b} \mathrm{~d} \hat{t} \\
& \leq C \delta^{(i-1 / p) a} \int_{t_{0}}^{t}\left\|\partial_{x}^{i} U(\hat{t})\right\|_{L^{p}}^{b} \mathrm{~d} \hat{t} .
\end{aligned}
$$


Substituting the above estimates for $R_{j},(j=1, \cdots, 4)$ into (3.3), we obtain (3.2). This completes the proof.

LEMMA3.2. Suppose that the assumptions of Proposition 2.4 hold. Then

$$
\begin{aligned}
& \left\|\varphi_{x}(t)\right\|_{ \pm}^{2}+\int_{t_{0}}^{t}\left\|\varphi_{x}\right\|_{ \pm}^{2}(\hat{t}) \mathrm{d} \hat{t} \\
& \leq C\left\{\left\|\left(\varphi_{x}, \psi\right)\left(t_{0}\right)\right\|_{ \pm}^{2}+\|\psi(t)\|_{ \pm}^{2}+\int_{t_{0}}^{t}\left\|\left(\phi_{x}, \psi_{x}, \psi_{x x}\right)\right\|_{ \pm}^{2}(\hat{t}) \mathrm{d} \hat{t}+\delta\right\} \\
& +C \varepsilon \int_{t_{0}}^{t}\left\|\left(\varphi_{x}, \psi_{x}, \psi_{x x}\right)\right\|_{ \pm}^{2}(\hat{t}) \mathrm{d} \hat{t} .
\end{aligned}
$$

PROOF. Multiplying the second equation of (2.9) by $-\frac{\varphi_{x}}{v}$, one obtains

$$
\begin{aligned}
& \left(\frac{1}{2}\left(\frac{\varphi_{x}}{v}\right)^{2}-\frac{\varphi_{x}}{v} \psi\right)_{t}+\left(\frac{\psi \psi_{x}}{v}\right)_{x}=\left(\frac{\psi_{x x} \varphi_{x}}{v^{2}}-\frac{\psi_{x} \varphi_{x}^{2}}{v^{3}}\right)-\left(\frac{U_{x} \varphi_{x}^{2}}{v^{3}}\right) \\
& +\left(\frac{\varphi_{x}^{2}}{v} p_{v}(v, \theta)+\frac{\psi_{x}^{2}}{v}\right)+\frac{\varphi_{x} \phi_{x}}{v} p_{\theta}(v, \theta)+\left(\frac{\psi \varphi_{x} U_{x}}{v^{2}}-\frac{\psi \psi_{x} V_{x}}{v^{2}}\right) \\
& +\frac{\varphi_{x}}{v}\left\{V_{x}\left(p_{v}(v, \theta)-p_{V}(V, \Theta)\right)+\Theta_{x}\left(p_{\theta}(v, \theta)-p_{\Theta}(V, \Theta)\right)\right\} \\
& +\varepsilon\left(-\frac{\varphi_{x} \psi_{x x}}{v^{2}}+\frac{\psi_{x} \varphi_{x}^{2}}{v^{3}}\right)+\varepsilon\left(-\frac{\varphi_{x} U_{x x}}{v^{2}}+\frac{\varphi_{x} U_{x} V_{x}}{v^{3}}\right)+\varepsilon\left(\frac{\psi_{x} \varphi_{x} V_{x}}{v^{3}}+\frac{\varphi_{x}^{2} U_{x}}{v^{3}}\right) .
\end{aligned}
$$

Integrating (3.5) with respect to $x, t$ over $\left(t_{0}, t\right) \times \mathbb{R}$, we infer $\left\|\varphi_{x}(t)\right\|_{ \pm}^{2}+\int_{t_{0}}^{t}\left\|\varphi_{x}\right\|_{ \pm}^{2}(\hat{t}) \mathrm{d} \hat{t}$

$$
\leq C\left\{\left\|\left(\varphi_{x}, \psi\right)\left(t_{0}\right)\right\|_{ \pm}^{2}+\|\psi(t)\|_{ \pm}^{2}+\int_{t_{0}}^{t}\left(\left\|\left(\psi_{x}, \phi_{x}\right)\right\|_{ \pm}^{2}+\left\|\sqrt{V_{t}}(\varphi, \phi)\right\|_{ \pm}^{2}\right)(\hat{t}) \mathrm{d} \hat{t}+\sum_{j=5}^{j=11} R_{j}\right\},
$$

with

$$
\begin{gathered}
R_{5}=-\int_{t_{0}}^{t} \int_{ \pm}\left(\frac{\psi \psi_{x}}{v}\right)_{x}(x, \hat{t}) \mathrm{d} x \mathrm{~d} \hat{t}, \\
R_{6}=\int_{t_{0}}^{t} \int_{ \pm}\left(\left|\psi U_{x} \varphi_{x}\right|+\left|V_{x} \psi \psi_{x}\right|\right)(x, \hat{t}) \mathrm{d} x \mathrm{~d} \hat{t}, \\
R_{7}=\int_{t_{0}}^{t} \int_{ \pm}\left(\left|\psi_{x x} \varphi_{x}\right|+\left|\psi_{x} \varphi_{x}^{2}\right|\right)(x, \hat{t}) \mathrm{d} x \mathrm{~d} \hat{t}, \\
R_{8}=\int_{t_{0}}^{t} \int_{ \pm}\left|U_{x} \varphi_{x}^{2}\right|(x, \hat{t}) \mathrm{d} x \mathrm{~d} \hat{t}, R_{9}=\varepsilon R_{7}, \\
R_{10}=\varepsilon \int_{t_{0}}^{t} \int_{ \pm}\left(\left|U_{x x} \varphi_{x}\right|+\left|U_{x} V_{x} \varphi_{x}\right|\right)(x, \hat{t}) \mathrm{d} x \mathrm{~d} \hat{t}, \\
R_{11}=\varepsilon \int_{t_{0}}^{t} \int_{ \pm}\left(\left|V_{x} \psi_{x} \varphi_{x}\right|+\left|U_{x} \varphi_{x}^{2}\right|\right)(x, \hat{t}) \mathrm{d} x \mathrm{~d} \hat{t},
\end{gathered}
$$

where $R_{j},(j=5, \cdots, 11)$ can be bounded as follows, using Sobolev's imbedding theorem and Lemma2.3 (iii) (see [25] for detail).

$$
\begin{array}{ll}
R_{5} \leq C \int_{t_{0}}^{t}\left\|\psi_{x}\right\|^{2}(\hat{t}) \mathrm{d} \hat{t}+C, & R_{6} \leq C \int_{t_{0}}^{t}\left\|\left(\varphi_{x}, \psi_{x}\right)\right\|^{2}(\hat{t}) \mathrm{d} \hat{t}+C, \\
R_{7} \leq C \int_{t_{0}}^{t}\left\|\left(\varphi_{x}, \psi_{x}, \psi_{x x}\right)\right\|^{2}(\hat{t}) \mathrm{d} \hat{t}+C, & R_{8} \leq C \int_{t_{0}}^{t}\left\|\varphi_{x}\right\|^{2}(\hat{t}) \mathrm{d} \hat{t}+C, \\
R_{9} \leq C \varepsilon \int_{t_{0}}^{t}\left\|\left(\varphi_{x}, \psi_{x}, \psi_{x x}\right)\right\|^{2}(\hat{t}) \mathrm{d} \hat{t}+C \varepsilon, & R_{10} \leq C \varepsilon \int_{t_{0}}^{t}\left\|\varphi_{x}\right\|^{2}(\hat{t}) \mathrm{d} \hat{t}+C \varepsilon, \\
R_{10} \leq C \varepsilon \int_{t_{0}}^{t}\left\|\left(\psi_{x}, \varphi_{x}\right)\right\|^{2}(\hat{t}) \mathrm{d} \hat{t}+C \varepsilon . &
\end{array}
$$


Inserting the estimates for $R_{j},(j=5, \cdots, 11)$ into (3.6), we arrive at

$$
\begin{aligned}
& \left\|\varphi_{x}(t)\right\|_{ \pm}^{2}+\int_{t_{0}}^{t}\left\|\varphi_{x}\right\|_{ \pm}^{2}(\hat{t}) \mathrm{d} \hat{t} \\
& \leq C\left\{\left\|\left(\varphi_{x}, \psi\right)\left(t_{0}\right)\right\|_{ \pm}^{2}+\|\psi(t)\|_{ \pm}^{2}+\int_{t_{0}}^{t}\left\|\left(\phi_{x}, \psi_{x}, \psi_{x x}\right)\right\|_{ \pm}^{2}(\hat{t}) \mathrm{d} \hat{t}+\delta\right\} \\
& \quad+C \varepsilon \int_{t_{0}}^{t}\left\|\left(\varphi_{x}, \psi_{x}, \psi_{x x}\right)\right\|_{ \pm}^{2}(\hat{t}) \mathrm{d} \hat{t} .
\end{aligned}
$$

Finally, combining Lemma 3.1 with Lemma 3.2, we conclude

$$
\begin{aligned}
& \left\|\left(\varphi, \psi, \phi, \varphi_{x}\right)(t)\right\|^{2}+\int_{t_{0}}^{t}\left(\left\|\sqrt{V_{t}}(\varphi, \phi)\right\|^{2}+\left\|\left(\varphi_{x}, \psi_{x}, \phi_{x}\right)\right\|_{ \pm}^{2}\right)(\hat{t}) \mathrm{d} \hat{t} \\
& \leq C\left\{\left\|(\varphi, \psi, \phi)\left(t_{0}\right)\right\|^{2}+\left\|\varphi_{x}(t)\right\|_{ \pm}^{2}+\int_{t_{0}}^{t}\left\|\psi_{x x}\right\|_{ \pm}^{2}(\hat{t}) \mathrm{d} \hat{t}+\delta\right\} \\
& \quad+C \varepsilon \int_{t_{0}}^{t}\left\|\left(\varphi_{x}, \phi_{x}, \psi_{x}, \psi_{x x}\right)\right\|_{ \pm}^{2}(\hat{t}) \mathrm{d} \hat{t}+C \kappa \int_{t_{0}}^{t}\left\|\left(\varphi_{x}, \phi_{x}\right)\right\|_{ \pm}^{2}(\hat{t}) \mathrm{d} \hat{t}+C \varepsilon .
\end{aligned}
$$

Comparing with the standard energy estimate for the compressible Navier-Stokes equations, we refer (3.8) to the basic energy estimate.

Next, we proceed to estimate higher order derivatives of $\psi, \phi$ in the space $L^{\infty}\left(t_{0}, t ; L^{2}\left(\mathbb{R}^{ \pm}\right)\right)$.

LEMMA 3.3.Suppose that the assumptions of Proposition 2.4 hold. Then,

$$
\begin{aligned}
& \left\|\psi_{x}(t)\right\|_{ \pm}^{2}+\int_{t_{0}}^{t}\left\|\psi_{x x}\right\|_{ \pm}^{2}(\hat{t}) \mathrm{d} \hat{t} \\
& \leq C\left\{\left\|(\varphi, \psi, \phi)\left(t_{0}\right)\right\|_{ \pm}^{2}+\left\|\psi_{x}\left(t_{0}\right)\right\|_{ \pm}^{2}\right\}+C \varepsilon \int_{t_{0}}^{t}\left\|\left(\varphi_{x}, \phi_{x}, \psi_{x}, \psi_{x x}\right)\right\|_{ \pm}^{2}(\hat{t}) \mathrm{d} \hat{t} \\
& \quad+C \kappa \int_{t_{0}}^{t}\left\|\left(\varphi_{x}, \phi_{x}\right)\right\|_{ \pm}^{2}(\hat{t}) \mathrm{d} \hat{t}+C \varepsilon .
\end{aligned}
$$

PROOF. Multiplying the second equation of (2.9) by $-\psi_{x x}$, one obtains

$$
\begin{aligned}
& \left(\frac{\psi_{x}^{2}}{2}\right)_{t}-\left(\psi_{t} \psi_{x}\right)_{x}+\varepsilon \frac{\psi_{x x}^{2}}{v} \\
& =\left(p_{v}(v, \theta) \varphi_{x}+p_{\theta}(v, \theta) \phi_{x}\right) \psi_{x x}+\varepsilon \frac{\varphi_{x} \psi_{x} \psi_{x x}}{v^{2}}+\varepsilon \frac{V_{x} \psi_{x} \psi_{x x}}{v^{2}} \\
& +\varepsilon \frac{U_{x} \varphi_{x} \psi_{x x}}{v^{2}}+V_{x}\left\{p_{v}(v, \theta)-p_{V}(V, \Theta)\right\} \psi_{x x} \\
& +\Theta_{x}\left\{p_{\theta}(v, \theta)-p_{\Theta}(V, \Theta)\right\} \psi_{x x}-\varepsilon \frac{U_{x x} \psi_{x x}}{v}+\varepsilon \frac{V_{x} U_{x} \psi_{x x}}{v^{2}},
\end{aligned}
$$

which, by integrating with respect to $x$ and $t$, leads to

$$
\begin{aligned}
& \left\|\psi_{x}(t)\right\|_{ \pm}^{2}+\varepsilon \int_{t_{0}}^{t}\left\|\psi_{x x}\right\|_{ \pm}^{2}(\hat{t}) \mathrm{d} \hat{t} \\
& \leq\left\|\psi_{x}\left(t_{0}\right)\right\|_{ \pm}^{2}+\int_{t_{0}}^{t} \int_{ \pm}\left(\psi_{t} \psi_{x}\right)_{x}(x, \hat{t}) \mathrm{d} x \mathrm{~d} \hat{t} \\
& \quad+C \int_{t_{0}}^{t}\left(\left\|\left(\varphi_{x}, \psi_{x}, \phi_{x}\right)\right\|_{ \pm}^{2}+\left\|\sqrt{V_{t}}(\varphi, \phi)\right\|^{2}\right)(\hat{t}) \mathrm{d} \hat{t} \\
& \quad+C \varepsilon \int_{t_{0}}^{t} \int_{ \pm}\left(\left|\varphi_{x} \psi_{x} \psi_{x x}\right|+\left|V_{x} \psi_{x} \psi_{x x}\right|+\left|U_{x} \varphi_{x} \psi_{x x}\right|\right)(x, \hat{t}) \mathrm{d} x \mathrm{~d} \hat{t} \\
& \quad+C \varepsilon \int_{t_{0}}^{t} \int_{ \pm}\left|\psi_{x x}\right|\left(\left|U_{x x}\right|+\left|U_{x} V_{x}\right|\right)(x, \hat{t}) \mathrm{d} x \mathrm{~d} \hat{t},
\end{aligned}
$$

The terms on the right hand side of (3.11) can be bounded as follows (see [25] 
for detail),

$$
\begin{aligned}
& \int_{t_{0}}^{t} \int_{ \pm}\left(\psi_{t} \psi_{x}\right)_{x}(x, \hat{t}) \mathrm{d} x \mathrm{~d} \hat{t} \leq C \int_{t_{0}}^{t}\left(\left\|\psi_{x x}\right\|+\|\varphi\|^{2}+\|\psi\|^{2}+\left\|\psi_{x}\right\|^{2}\right)(\hat{t}) \mathrm{d} \hat{t}, \\
& \varepsilon \int_{t_{0}}^{t} \int_{ \pm}\left|\left(\varphi_{x} \psi_{x} \psi_{x x}\right)\right|(x, \hat{t}) \mathrm{d} x \mathrm{~d} \hat{t} \leq C \varepsilon \int_{t_{0}}^{t}\left(\left\|\psi_{x x}\right\|_{ \pm}^{2}+N\left(t_{0}, t\right)\left\|\psi_{x}\right\|\right)(\hat{t}) \mathrm{d} \hat{t}, \\
& \varepsilon \int_{t_{0}}^{t} \int_{ \pm}\left|\psi_{x x}\right|\left(\left|V_{x} \psi_{x}\right|+\left|U_{x} \varphi_{x}\right|\right)(x, \hat{t}) \mathrm{d} x \mathrm{~d} \hat{t} \leq C \varepsilon \int_{t_{0}}^{t}\left(\left\|\psi_{x x}\right\|_{ \pm}^{2}+\delta\left\|\left(\varphi_{x}, \psi_{x}\right)\right\|\right)(\hat{t}) \mathrm{d} \hat{t}, \\
& \varepsilon \int_{t_{0}}^{t} \int_{ \pm}\left|\psi_{x x}\right|\left(\left|U_{x x}\right|+\left|U_{x} V_{x}\right|\right)(x, \hat{t}) \mathrm{d} x \mathrm{~d} \hat{t} \leq C \varepsilon \int_{t_{0}}^{t} \|\left.\psi_{x x}\right|_{ \pm} ^{2}(\hat{t}) \mathrm{d} \hat{t}+C \varepsilon \delta .
\end{aligned}
$$

Substituting the above estimates into (3.11), we obtain (3.9).

Similarly, we can bound the derivatives of $\phi$ as follows.

LEMMA 3.4Assume that the assumptions of Proposition 2.4 hold. Then,

$$
\begin{aligned}
& \left\|\phi_{x}(t)\right\|_{ \pm}^{2}+\int_{t_{0}}^{t}\left\|\phi_{x x}\right\|_{ \pm}^{2}(\hat{t}) \mathrm{d} \hat{t} \\
& \leq C\left(\left\|(\varphi, \psi, \phi)\left(t_{0}\right)\right\|+\left\|\phi_{x}\left(t_{0}\right)\right\|_{ \pm}^{2}+\delta\right) \\
& \quad+C \varepsilon \int_{t_{0}}^{t}\left(\left\|\left(\phi_{x x}, \psi_{x}, \psi_{x x}\right)\right\|_{ \pm}^{2}+\left\|\psi_{x}\right\|_{ \pm}\right)(\hat{t}) \mathrm{d} \hat{t} \\
& \quad+C \kappa \int_{t_{0}}^{t}\left(\left\|\left(\varphi_{x}, \phi_{x}\right)\right\|_{ \pm}+\left\|\left(\phi_{x}, \phi_{x x}\right)\right\|_{ \pm}^{2}\right)(\hat{t}) \mathrm{d} \hat{t}+C \varepsilon+C \kappa .
\end{aligned}
$$

PROOF. Multiplying the third equation of (2.9) by $-\phi_{x x}$, then integrating with respect to $x$ and $t$, utilizing (3.7) and (3.8), we deduce that

$$
\begin{aligned}
& \left\|\phi_{x}(t)\right\|_{ \pm}^{2}+\int_{t_{0}}^{t}\left\|\phi_{x x}\right\|_{ \pm}^{2}(\hat{t}) \mathrm{d} \hat{t} \\
& \leq \mid \phi_{x}\left(t_{0}\right)\left\|_{ \pm}^{2}+C \varepsilon \int_{t_{0}}^{t}\right\| \psi_{x} \|_{ \pm}^{2}(\hat{t}) d \hat{t}+C \int_{t_{0}}^{t} \int_{ \pm}\left(\phi_{t} \phi_{x}\right)_{x}(x, \hat{t}) \mathrm{d} x \mathrm{~d} \hat{t} \\
& +C \kappa \int_{t_{0}}^{t} \int_{ \pm}\left|\phi_{x x}\right|\left|\varphi_{x} \phi_{x}\right|(x, \hat{t}) \mathrm{d} x \mathrm{~d} \hat{t}+C \varepsilon \int_{t_{0}}^{t} \int_{ \pm}\left|\phi_{x x}\right|\left|\psi_{x}^{2}\right|(x, \hat{t}) \mathrm{d} x \mathrm{~d} \hat{t} \\
& +C \int_{t_{0}}^{t} \int_{ \pm}\left|U_{x} \phi_{x x}\right|(|\varphi|+|\phi|)(x, \hat{t}) \mathrm{d} x \mathrm{~d} \hat{t} \\
& +C \kappa \int_{t_{0}}^{t} \int_{ \pm}\left|\phi_{x x}\right|\left(\left|V_{x} \phi_{x}\right|+\left|\Theta_{x} \varphi_{x}\right|\right)(x, \hat{t}) \mathrm{d} x \mathrm{~d} \hat{t} \\
& +C \varepsilon \int_{t_{0}}^{t} \int_{ \pm}\left|\phi_{x x}\right|\left|\psi_{x} U_{x}\right|(x, \hat{t}) \mathrm{d} x \mathrm{~d} \hat{t}+C \varepsilon \int_{t_{0}}^{t} \int_{ \pm}\left|\phi_{x x}\right|\left|U_{x}^{2}\right|(x, \hat{t}) \mathrm{d} x \mathrm{~d} \hat{t} \\
& +C \kappa \int_{t_{0}}^{t} \int_{ \pm}\left|\phi_{x x}\right|\left(\left|\Theta_{x} V_{x}\right|+\left|\Theta_{x x}\right|\right)(x, \hat{t}) \mathrm{d} x \mathrm{~d} \hat{t},
\end{aligned}
$$

where the right hand side can be estimated as follows (see [25] for detail),

$$
\begin{gathered}
\int_{t_{0}}^{t} \int_{ \pm}\left(\phi_{t} \phi_{x}\right)_{x} \mathrm{~d} x \mathrm{~d} \hat{t} \leq \frac{1}{16} \int_{t_{0}}^{t}\left\|\phi_{x x}\right\|_{ \pm}^{2}(\hat{t}) \mathrm{d} \hat{t}+C \int_{t_{0}}^{t}\left(\|\psi\|^{2}+\|\phi\|^{2}+\left\|\phi_{x}\right\|_{ \pm}^{2}\right)(\hat{t}) \mathrm{d} \hat{t} \\
\kappa \int_{t_{0}}^{t} \int_{ \pm}\left|\varphi_{x} \phi_{x} \phi_{x x}\right|(x, \hat{t}) \mathrm{d} x \mathrm{~d} \hat{t} \leq \frac{\kappa}{16} \int_{t_{0}}^{t}\left\|\left.\phi_{x x}\right|_{ \pm} ^{2}(\hat{t}) \mathrm{d} \hat{t}+C \kappa N\left(t_{0}, t\right) \int_{t_{0}}^{t}\right\| \phi_{x} \|_{ \pm}^{2}(\hat{t}) \mathrm{d} \hat{t} \\
\varepsilon \int_{t_{0}}^{t} \int_{ \pm}\left|\psi_{x}^{2} \phi_{x x}\right|(x, \hat{t}) \mathrm{d} x \mathrm{~d} \hat{t} \\
\leq \frac{\varepsilon}{16} \int_{t_{0}}^{t}\left\|\phi_{x x}\right\|_{ \pm}^{2}(\hat{t}) \mathrm{d} \hat{t}+C \varepsilon N\left(t_{0}, t\right) \int_{t_{0}}^{t}\left(\left\|\psi_{x x}\right\|_{ \pm}^{2}+\left\|\psi_{x}\right\|_{ \pm}^{2}\right)(\hat{t}) \mathrm{d} \hat{t} \\
\int_{t_{0}}^{t} \int_{ \pm}\left|U_{x} \phi_{x x}\right|(|\varphi|+|\phi|)(x, \hat{t}) \mathrm{d} x \mathrm{~d} \hat{t} \\
\leq \frac{1}{16} \int_{t_{0}}^{t}\left\|\phi_{x x}\right\|_{ \pm}^{2}(\hat{t}) \mathrm{d} \hat{t}+C \delta \int_{t_{0}}^{t}\left\|\sqrt{V_{t}}(\varphi, \phi)\right\|(\hat{t}) \mathrm{d} \hat{t}
\end{gathered}
$$




$$
\begin{gathered}
\kappa \int_{t_{0}}^{t} \int_{ \pm}\left|\phi_{x x}\right|\left(\left|V_{x} \phi_{x}\right|+\left|\Theta_{x} \varphi_{x}\right|\right)(x, \hat{t}) \mathrm{d} x \mathrm{~d} \hat{t} \\
\leq \frac{\kappa}{16} \int_{t_{0}}^{t}\left\|\phi_{x x}\right\|_{ \pm}^{2}(\hat{t}) \mathrm{d} \hat{t}+C \kappa \delta \int_{t_{0}}^{t}\left\|\left(\varphi_{x}, \phi_{x}\right)\right\|(\hat{t}) \mathrm{d} \hat{t} \\
\varepsilon \int_{t_{0}}^{t} \int_{ \pm}\left|\phi_{x x}\right|\left|U_{x} \psi_{x}\right|(x, \hat{t}) \mathrm{d} x \mathrm{~d} \hat{t} \leq \frac{\varepsilon}{16} \int_{t_{0}}^{t}\left\|\phi_{x x}\right\|_{ \pm}^{2}(\hat{t}) \mathrm{d} \hat{t}+C \varepsilon \delta \int_{t_{0}}^{t}\left\|\psi_{x}\right\|(\hat{t}) \mathrm{d} \hat{t}, \\
\varepsilon \int_{t_{0}}^{t} \int_{ \pm}\left|\phi_{x x}\right|\left|U_{x}^{2}\right|(x, \hat{t}) \mathrm{d} x \mathrm{~d} \hat{t} \leq \frac{\varepsilon}{16} \int_{t_{0}}^{t}\left\|\phi_{x x}\right\|_{ \pm}^{2}(\hat{t}) \mathrm{d} \hat{t}+C \varepsilon \delta^{1 / 4},
\end{gathered}
$$

and

$$
\kappa \int_{t_{0}}^{t} \int_{ \pm}\left|\phi_{x x}\right|\left(\left|\Theta_{x} V_{x}\right|+\left|\Theta_{x x}\right|\right)(x, \hat{t}) \mathrm{d} x \mathrm{~d} \hat{t} \leq \frac{\kappa}{16} \int_{t_{0}}^{t}\left\|\phi_{x x}\right\|_{ \pm}^{2}(\hat{t}) \mathrm{d} \hat{t}+C \kappa \delta^{1 / 4}
$$

Substitution of the above estimates into (3.13) gives Lemma 3.4 immediately.

Now, combining Lemma 3.1 - 3.4, we obtain Proposition 2.4.

\section{Conflicts of Interest}

The authors declare no conflicts of interest regarding the publication of this paper.

\section{References}

[1] Di Perna, R.J. (1983) Convergence of Approximate Solutions to Conservation Laws. Archive for Rational Mechanics and Analysis, 83, 27-70. https://doi.org/10.1007/BF00251724

[2] Hoff, D. and Liu, T.P. (1989) The Inviscid Limit for Navier-STOKES equations of Compressible Isentropic Flow with Shock Data. Indiana University Mathematics Journal, 38, 861-915. https://doi.org/10.1512/iumj.1989.38.38041

[3] Goodman, J. and Xin, Z. (1992) Viscous Limits for Piecewise Smooth Solutions to Systems of Conservation Laws. Archive for Rational Mechanics and Analysis, 121, 235-265. https://doi.org/10.1007/BF00410614

[4] Xin, Z. (1993) Zero Dissipation Limit to Rarefaction Waves for the One-Dimensional Navier-Stokes Equations of Compressible Isentropic Gases. Communications on Pure and Applied Mathematics, 46, 621-665. https://doi.org/10.1002/cpa.3160460502

[5] Xin, Z. (1998) Viscous Boundary Layers and Their Stability (I). Journal of Partial Differential Equations, 11, 97-124.

[6] Xin, Z. and Yanagisawa, T. (1999) Zero-Viscosity Limit of the Linearized Navier-Stokes Equations for a Compressible Viscous Fluid in the Half-Plane. Communications on Pure and Applied Mathematics, 52, 479-541. https://doi.org/10.1002/(SICI)1097-0312(199904)52:4<479::AID-CPA4>3.0.CO;2-1

[7] Wang, Y. and Xin, Z. (2005) Zero-Viscosity Limit of the Linearized Compressible Navier-Stokes Equations with Highly Oscillatory Forces in the Half-Plane. SIAM Journal on Mathematical Analysis, 37, 1256-1298. https://doi.org/10.1137/040614967

[8] Serre, D. and Zumbrun, K. (2001) Boundary Layer Stability in Real Vanishing Viscosity Limit. Communications in Mathematical Physics, 221, 267-292. https://doi.org/10.1007/s002200100486 
[9] Rousset, F. (2003) Stability of Small Amplitude Boundary Layers for Mixed Hyperbolic-Parabolic Systems. Transaction of American Mathematical Society, 355, 2991-3008. https://doi.org/10.1090/S0002-9947-03-03279-3

[10] DiPerna, R.J. (1983) Convergence of the Viscosity Method for Isentropic Gas Dynamics. Communications in Mathematical Physics, 91, 1-30. https://doi.org/10.1007/BF01206047

[11] Lions, P.L., Perthame, B. and Tadmor, E. (1994) Kinetic Formulation of the Isentropic Gas Dynamics and P-System. Communications in Mathematical Physics, 163, 415-431. https://doi.org/10.1007/BF02102014

[12] Lions, P.L., Perthame, B. and Souganidis, P.E. (1996) Existence and Stability of Entropy Solutions for the Hyperbolic Systems of Isentropic Gas Dynamics in Eulerian and Lagrangian Coordinates. Communications on Pure and Applied Mathematics, 49, 599-638. https://doi.org/10.1002/(SICI)1097-0312(199606)49:6<599::AID-CPA2>3.0.CO;2-5

[13] Yu, S. (1999) Zero-Dissipation Limit of Solutions with Shocks for Systems of Hyperbolic Conservation Laws. Archive for Rational Mechanics and Analysis, 146, 275-370. https://doi.org/10.1007/s002050050143

[14] Grenier, E. and Rousset, F. (2001) Stability of One-Dimensional Boundary Layers by Using Green's Functions. Communications on Pure and Applied Mathematics, 54, 1343-1385. https://doi.org/10.1002/cpa.10006

[15] Serre, D. (2001) Sur la stabilité des couches limites de viscosité. Annales de l'Institut Fourier, 51, 109-129. https://doi.org/10.5802/aif.1818

[16] Bressan, A. and Yang, T. (2004) On the Convergence Rate of Vanishing Viscosity Approximations. Communications on Pure and Applied Mathematics, 57, 1075-1109. https://doi.org/10.1002/cpa.20030

[17] Gues, O., Metivier, G., Williams, M. and Zumbrun, K. (2004) Multidimensional Viscous Shocks II: The Small Viscosity Limit. Communications on Pure and Applied Mathematics, 57, 141-218.

[18] Gues, O., Metivier, G., Williams, M. and Zumbrun, K. (2004) A New Approach to the Stability of Multidimensional Viscous Shocks.

[19] Bianchini, S. and Bressan, A. (2005) Vanishing Viscosity Solutions of Nonlinear Hyperbolic Systems. Annals of Mathematics, 161, 223-342.

https://doi.org/10.4007/annals.2005.161.223

[20] Bressan, A. (2000) BV-Solutions to Hyperbolic Systems by Vanishing Viscosity. S.I.S.S.A., Trieste.

[21] Dafermos, C.M. (2000) Hyperbolic Conservation Laws in Continuum Physics. Springer-Verlag, Berlin, Heidelberg. https://doi.org/10.1007/978-3-662-22019-1

[22] Serre, D. (2000) Systems of Conservation Laws I, II. Cambridge Univ. Press, Cambridge.

[23] Wang, W.C. and Xin, Z. (1998) Fluid-Dynamic Limit for the Centered Rarefaction Wave of the Broadwall Equation. Journal of Differential Equations, 150, 438-461. https://doi.org/10.1006/jdeq.1998.3488

[24] Hsiao, L. and Pan, R.H. (1999) Zero Relaxation Limit to Centered Rarefaction Waves for a Rate-Type Viscoelastic System. Journal of Differential Equations, 157, 20-40. https://doi.org/10.1006/jdeq.1998.3615

[25] Jiang, S., Ni, G. and Sun, W. (2012) Vanishing Viscosity Limit to Rarefaction Waves for the Navier-Stokes Equations of One-Dimensional Compressible Heat-Conducting Fluids. SIAM Journal on Mathematical Analysis, 38, 368-384. 
[26] Smoller, J. (1983) Shock Waves and Reactive Diffusion Equations. Springer-Verlag, New York, Berlin, Heidelberg. https://doi.org/10.1007/978-1-4684-0152-3

[27] Courant, R. and Friedrichs, K.O. (1948) Supersonic Flows and Shock Waves. Wiley-Interscience, New York.

[28] Hoff, D. (1992) Global Well-Posedness of Cauchy Problem for Navier-Stokes Equations of Nonisentropic Flow with Discontinuous Initial Data. Journal of Differential Equations, 95, 33-74. https://doi.org/10.1016/0022-0396(92)90042-L 